\title{
Microstructural Evolution During Hot Rolling of an AZ31 Mg Alloy
}

\author{
J. A. del Valle ${ }^{1,2}$, M.T. Pérez-Prado ${ }^{1}$, and O.A. Ruano ${ }^{1}$ \\ ${ }^{1}$ Dept. of Physical Metallurgy, Centro Nacional de Investigaciones Metalúrgicas (CENIM), \\ CSIC, Avda. de Gregorio del Amo, 8, 28040 Madrid, Spain \\ ${ }^{2}$ Consejo Nacional de Investigaciones Científicas y Técnicas (CONICET), Argentina
}

Keywords: Texture, AZ31 Mg alloy, unidirectional rolling, cross rolling, recrystallization, twinning.

\begin{abstract}
The microstructural evolution of a AZ31 $\mathrm{Mg}$ alloy during hot rolling has been investigated using optical microscopy and texture (macro and micro) analysis as the main characterization tools. In particular, the differences between the microstructure obtained by unidirectional rolling (UR) and cross rolling (CR) are studied. Significant twinning activity is observed in both cases. Additionally, after cross rolling, a rather heterogeneous microstructure develops, with scattered regions populated by very fine grains. The strong basal fiber texture of the as-received material remains present after both hot rolling schemes. The imposibility to obtain accurate EBSD measurements within the twinned regions suggests that significant localized deformation takes place in those areas. Thus, due to the increase in the local strain energy, these regions become preferential sites for nucleation of recrystallization.
\end{abstract}

\section{Introduction}

Magnesium alloys are attractive materials due to their excellent specific properties such as low density $\left(1.74 \mathrm{~g} / \mathrm{cm}^{3}\right)$ and high specific strength that make them potentially suitable alloys for the transport industry [1]. It is also envisioned that $\mathrm{Mg}$ will play an important role in lightweight construction in the future [2]. However, $\mathrm{Mg}$ alloys exhibit limited ductility due to their hcp structure. Important efforts are devoted to overcome this difficulty.

The microstructure and mechanical properties of a given material are highly dependent on their previous thermomechanical processing [3]. In particular, during hot rolling, conditions such as rolling temperature, rolling direction and reduction per pass influence the operative deformation mechanisms both during rolling and during subsequent tensile testing of the rolled material by determining microstructural parameters such as grain size, dislocation density, size of second phase particles and others. Therefore, properties such as ductility and flow strength will also likely depend on the rolling conditions. The relationship between processing, microstructure and mechanical properties is still not thoroughly established for Mg alloys [4].

The aim of this work is to study the microstructural evolution of an AZ31 Mg alloy during unidirectional and cross rolling. Special emphasis is placed in investigating the macro- and microtexture development. The underlying microscopic mechanisms are investigated on the light of these findings.

\section{Experimental procedure}

The AZ31B Mg alloy studied was provided by Magnesium Elektron in the form of a 3mm-thick rolled sheet. The main alloying elements are $\mathrm{Al}(3 \%)$ and $\mathrm{Zn} \mathrm{(1 \% ).} \mathrm{In} \mathrm{the} \mathrm{as-received} \mathrm{state,} \mathrm{the}$ material is fully recrystallized (AZ31B-O). 
Hot rolling of the as-received material was performed at $375^{\circ} \mathrm{C}$. Both unidirectional rolling (UR) (6 passes of $0.25 \mathrm{~mm}$ reduction per pass) and cross rolling (CR)(6 passes with alternate perpendicular rolling direction and $0.25 \mathrm{~mm}$ per pass) were utilized. Tensile specimens of $20 \mathrm{~mm}$ gage length and a radius of 3 were machined out of the rolled sheet both with the tensile axis parallel and perpendicular to the rolling direction of the final pass. Tensile tests to failure were performed at $375^{\circ} \mathrm{C}$ and at a constant crosshead speed, resulting in initial strain rates of $1 \times 10^{-2} \mathrm{~s}^{-1}$ to $1 \times 10^{-3} \mathrm{~s}^{-1}$.

The microstructure of the AZ31 alloy was investigated by optical microscopy (OM) in the asreceived condition, after unidirectional and cross rolling, and after tensile testing under all the above mentioned conditions. Sample preparation consisted on grinding on SiC paper with increasingly finer grits, followed by mechanical polishing with $6 \mu \mathrm{m}$ and $1 \mu \mathrm{m}$ diamond paste and final polishing using colloidal silica. The grain structure was revealed by subsequent etching using a solution of ethanol $(100 \mathrm{ml})$, picric acid $(5 \mathrm{~g})$, acetic acid $(5 \mathrm{ml})$ and water $(10 \mathrm{ml})$. After chemical etching a residual layer remained in the sample surface that was eliminated by immersion in boiling ethanol.

Texture analysis has proven to be a powerful tool to obtain exhaustive microstructural information [5]. The macrotexture of the AZ31 alloy both as-received and after unidirectional and cross hot rolling was measured by the Schulz reflection method in a Siemens diffractometer furnished with a closed eulerian cradle. Five pole figures were measured, namely (100), (002), (110), (101), and (102). From them, recalculated pole figures were obtained using the standard spherical harmonics series expansion method. The microtexture of the material in the as-received condition as well as after hot rolling was also investigated. Microtexture measurements were performed by electron backscattered diffraction (EBSD) using the Oxford Instruments INCA (Crystal) software. Measurements were carried out in the as-received and processed samples in a JEOL SEM operating at $20 \mathrm{kV}$. Sample preparation for EBSD is difficult since, due to the low density of $\mathrm{Mg}$, Kikuchi diffraction patterns have very low intensity. Therefore a good surface preparation is crucial. Sample preparation consisted on grinding using SiC papers with 400, 600, and 1200 grit size. Final mechanical polishing was achieved with a $0.05 \mu \mathrm{m}$ alumina solution. Electropolishing was carried out with a solution of $40 \%$ ortophosphoric acid and $60 \%$ ethanol at $2{ }^{\circ} \mathrm{C}$ and using a voltage of $2-3$ volts. Finally, samples were cleaned with cold ethanol and dried with Ar flow.

\section{Results and discussion}

Figure 1 illustrates the microsctructure of the AZ31 alloy in the as-received condition (Fig.1a), after UR (Fig. 1b), and CR (Fig.1c). Initially, the material is fully recrystallized and possesses a rather heterogeneous grain size distribution, as can be observed in Fig.1a. On average, grain size is equal to $32 \mu \mathrm{m}$. After both unidirectional and cross rolling, the grain size decreases significantly, reaching a value of approximately $16 \mu \mathrm{m}$. Significant twinning seems to take place during rolling. Narrow twins form and they tend to align perpendicular to the rolling direction. This can be clearly seen in Fig. 1b, where the rolling direction is the horizontal. Cross rolling leads thus to a wider spread of the twins with respect to the rolling direction (Fig.1c). A highly heterogeneous microstructure develops. An enlarged view of the microstructure of the CR sample is shown in Fig.1d. Newly formed small grains, approximately $3 \mu \mathrm{m}$ in size, can be apreciated in specific scattered zones of the sample. Close inspection of the optical micrograph reveals that these small grains tend to nucleate preferentially in the twinned and grain boundary areas. However, in other areas of the CR sample, the onset of recrystallization was not observed.

Figure 2 shows the X-ray macrotexture corresponding to the AZ31 alloy in the as-received condition (Fig.2a) and after UR (Fig.2b) and CR (Fig.2c). The initial texture is a basal fiber texture, i.e., most of the grains have the c-axis perpendicular to the rolling plane. This texture is typical of $\mathrm{Mg}$ laminates [6]. No significant texture changes were observed after both rolling schemes in spite of the significant fraction of twinned material observed in the optical micrographs of Fig.1. The basal fiber texture remains present with almost no variation in its intensity. 
(a)

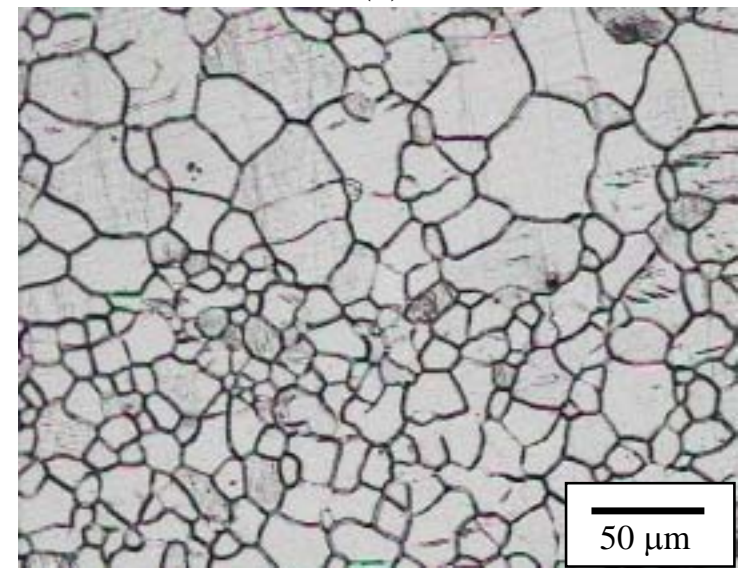

(c)

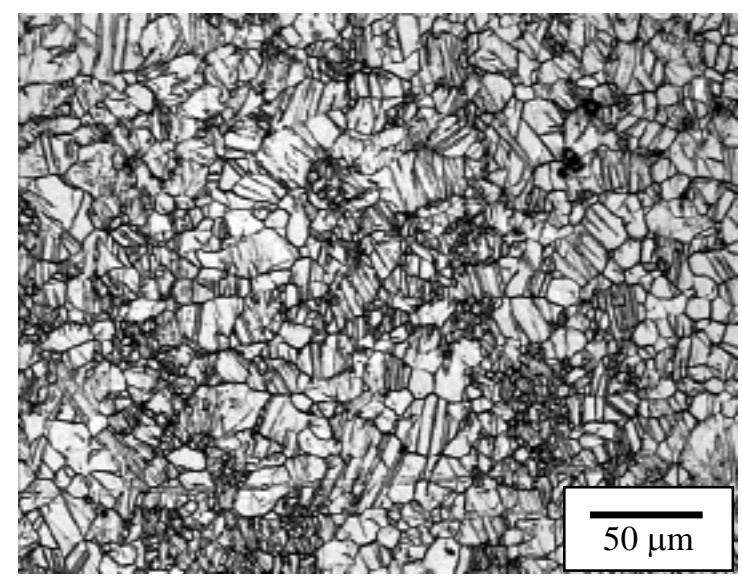

(b)

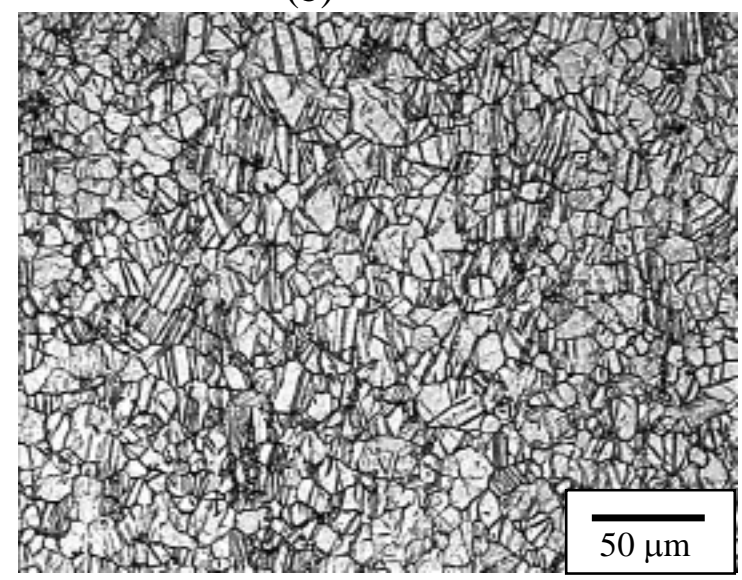

(d)

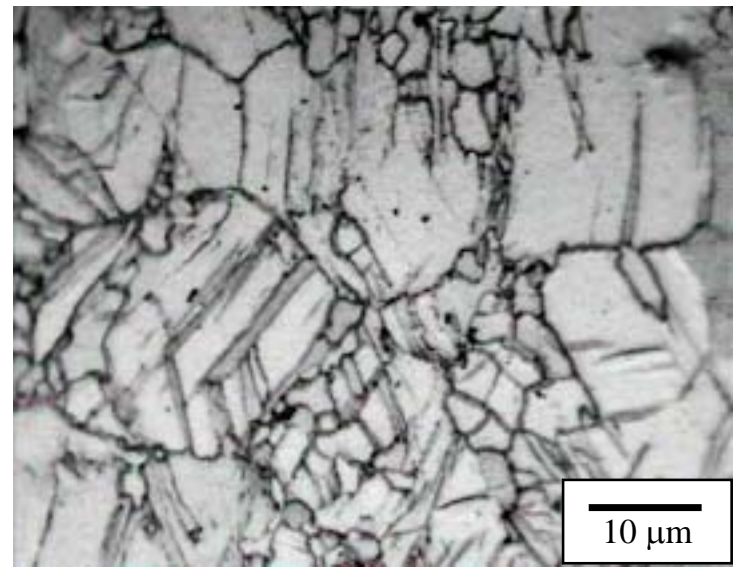

Figure 1. Optical micrographs showing the microstructure of the AZ31 alloy (a) in the as-received condition, (b)unidirectionally rolled (UR) at $375^{\circ} \mathrm{C}$, and (c) cross rolled (CR) at $375^{\circ} \mathrm{C}$. (d) Enlarged view of the heterogeneous microstructure developed after cross rolling at $375^{\circ} \mathrm{C}$.

(a)

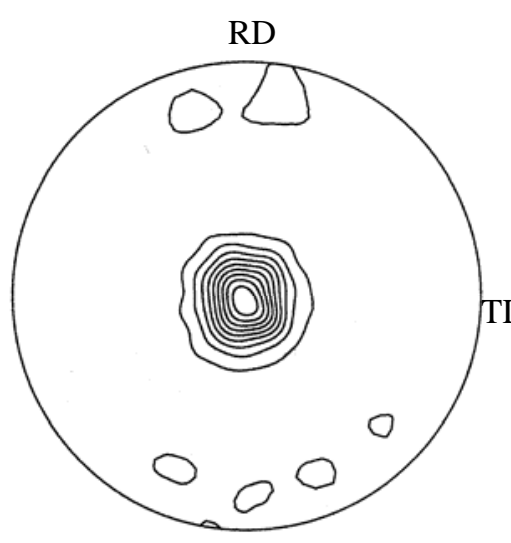

(b)

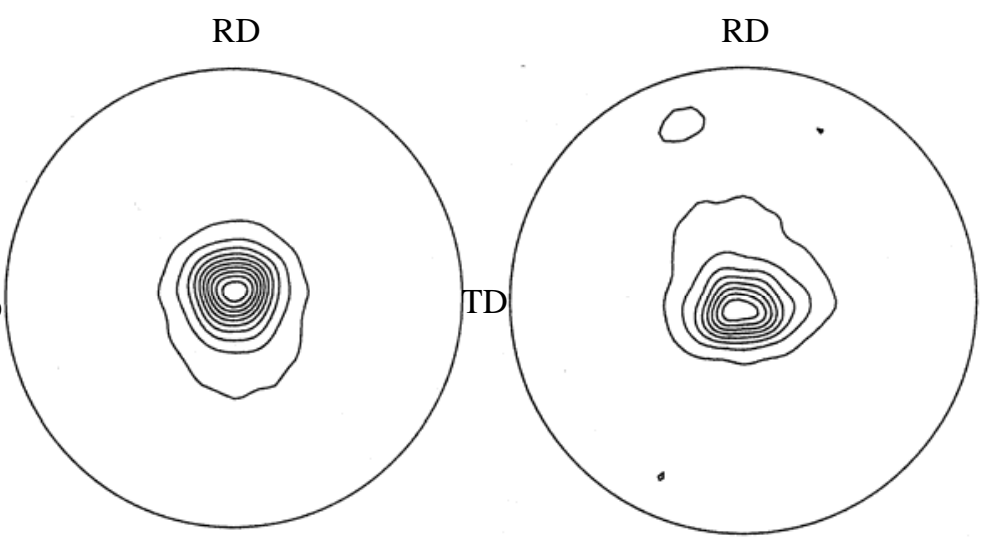

(c)
Figure 2. (0002) X-ray pole figures illustrating the macrotexture of the AZ31 alloy (a) in the asreceived condition, (b)unidirectionally rolled (UR) at $375^{\circ} \mathrm{C}$, and (c) cross rolled (CR) at $375^{\circ} \mathrm{C}$. $\mathrm{RD}$ denotes the rolling direction corresponding to the last rolling pass. Intensity levels: $1,2,3,4,5,6,7,8,9,10$. 
(a)



(b)

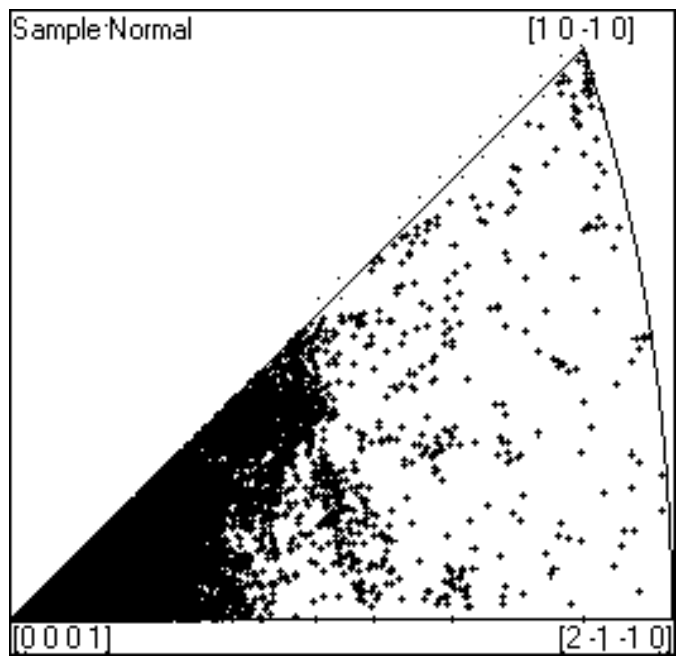

Figure 3. Microtexture of the unidirectionally rolled (UR) AZ31 alloy. (a) Quality map of a section parallel to the rolling plane; (b) Inverse pole figure showing the orientation of the normal direction (ND) in the area shown in (a).

(a)



(b)



Figure 4. Microtexture of the cross rolled (CR) AZ31 alloy. (a) Quality map of a section parallel to the rolling plane; (b) Inverse pole figure showing the orientation of the normal direction (ND) in the area shown in (a).

Figure 3 depicts the results obtained by EBSD examination of the rolled AZ31 alloy. Figure 3a is a quality map corresponding to a section of the UR sample parallel to the rolling plane. Figure $3 \mathrm{~b}$ illustrates the corresponding inverse pole figure showing the orientation of the normal direction in the same area. The microtexture measurements are overall consistent with the macrotexture measurements obtained by X-ray diffraction. It can be inferred from Fig.3 that a strong basal fiber texture remains present after hot rolling. However, black straight lines inside the grains are apparent in Fig. 3a, indicating regions where it was not possible to obtain reliable EBSD measurements. The morphology of these areas resembles the twin structure present after deformation observed by optical microscopy (Fig.1). Specifically, it can be seen that they tend to be aligned perpendicular to the rolling direction, which is the horizontal. Kikuchi patters could also not be resolved in the areas close to grain boundaries. The microtexture data corresponding to the CR sample is illustrated in Fig.4. Both a quality map taken along the rolling plane (Fig. 4a) as well as the corresponding inverse pole figure (Fig. 4b) showing the orientation of the normal direction are depicted. Again, consistently with the macrotexture measurements, a strong basal fiber texture is apparent. Kikuchi 
patterns could not be resolved within most of the twinned areas (black areas in Fig. 4a) and close to grain boundaries.

Several factors may cause Kikuchi patterns to become more diffuse at a specific location in the sample: bad sample surface preparation, the presence of a very fine structure (finer than the electron beam size), or a high lattice distorsion due to localized deformation. Surface preparation can be ruled out since good quality patterns are obtained in large areas of the sample and since the morphology of the regions that are not resolved resembles both the twin and grain boundary areas. Instead, we propose that heterogeneous deformation takes place during hot rolling, in such a way that localized deformation concentrates within the twins and along grain boundaries and thus diffuse patterns are obtained in these zones. The appearance of narrow twins in deformed polycrystalline $\mathrm{Mg}$ specimens has been previously reported [7]. It has been suggested that these twins may be significantly deformed by slip or second-order twinning shortly after they have formed. The present results agree with this observation but do not allow to infer whether the high localized deformation within the twins is caused by one or the other mechanism. The localization of deformation along grain boundary areas has also been recently observed by del Valle et al. [8] in a severely rolled AZ61 alloy. We suggest that, due to the increase in strain energy in the twin and grain boundary areas, these become preferential sites for the nucleation of recrystallization, as can be observed in Fig. 1d.

Preliminary tensile tests at $375^{\circ} \mathrm{C}$ and at strain rates of $1 \times 10^{-3} \mathrm{~s}^{-1}$ and $1 \times 10^{-2} \mathrm{~s}^{-1}$ are showing that the highest ductility is always achieved in the unidirectionally rolled material when tested with the tensile axis parallel to the rolling direction. Further work is being carried out to ellucidate the microscopic mechanisms responsible for the tensile defomation of the rolled AZ31 alloy as well as the influence of the heterogeneous microstructure resulting from rolling on the mechanical behavior.

\section{Conclusions}

The texture and microstructural evolution of a recrystallized AZ31 alloy during unidirectional and cross rolling has been investigated by optical microscopy, X-ray diffraction and EBSD. It has been found that, together with significant grain refinement, extensive twinning activity takes place during rolling. The microstructure of the cross rolled material seems to be more heterogeneous than that of the unidirectionally rolled alloy, with clusters of fine grains scattered throughout the microstructure. The as-received strong basal fiber texture is retained after deformation. The diffuseness of the Kikuchi patterns in the twinned and grain boundary areas is attributed to localized deformation within these zones. Thus, due to the increase in the local strain energy, these regions become favourable sites for the nucleation of recrystallization.

\section{Acknowledgements}

Special thanks are given to C. Moreno and J.A. Jiménez for assistance with the macrotexture measurements. MTP acknowledges a Ramón y Cajal 2001 contract, awarded by the Spanish Ministry of Science and Technology. Financial support from a CICYT grant MAT is appreciated. JAV is thankful to CONICET for a postdoctoral grant.

\section{References}

[1] B. L. Mordike and T. Ebert: Mater. Sci. Eng. A, vol. 302 (2001), p. 37.

[2] C. Jaschik, H. Haferkamp, M. Niemeyer: Magnesium Alloys and Their Applications (WileyVCH, Weinheim, Germany, 2000), p.41. 
[3] F. J. Humphreys and M. Hatherly: Recrystallization and Related Annealing Phenomena (Pergamon Press, United Kingdom, 1995).

[4] S. R. Agnew, M. H. Yoo, and C. N. Tomé: Acta mater., vol. 49 (2001), p.4277.

[5] V. Randle and O. Engler: Macrotexture, Microtexture and Orientation Mapping (Gordon and Breach Science Publishers, Amsterdam, The Netherlands, 2000).

[6] C. S. Roberts: Magnesium and its Alloys (John Wiley \& Sons, New York, 1960).

[7] F. E. Hauser, C. D. Starr, L. S. Tietz, and J. E. Dorn: Trans. ASM, vol. 47 (1955), p.102.

[8] J. A. del Valle, M. T. Pérez-Prado and O. A. Ruano: Mater. Sci. Eng. A, (2003) in press. 